\title{
Electricity peak demand classification with artificial neural networks
}

\author{
Krzysztof Gajowniczek, Rafik Nafkha, Tomasz Ząbkowski \\ Department of Informatics, Warsaw University of Life Sciences, \\ Nowoursynowska 159, 02-776 Warsaw, Poland \\ Email: \{krzysztof_gajowniczek, rafik_nafkha, tomasz_zabkowski\}@sggw.pl
}

\begin{abstract}
Demand peaks in electrical power system cause serious challenges for energy providers as these events are typically difficult to foresee and require the grid to support extraordinary consumption levels. Accurate peak forecasting enables utility providers to plan the resources and also to take control actions to balance electricity supply and demand. However, this is difficult in practice as it requires precision in prediction of peaks in advance. In this paper, our contribution is the proposal of data mining scheme to detect the peak load in the electricity system at country level. For this purpose we undertake the approach different from time series forecasting and represent it as pattern recognition problem. We utilize set of artificial neural networks to benefit from accurate detection of the peaks in the Polish power system. The key finding is that the algorithms can accurately detect $96.2 \%$ of the electricity peaks up to 24 hours ahead.
\end{abstract}

\section{INTRODUCTION}

E LECTRICITY consumption peaks appear in the electricity system as a consequence of collective behavior of end users which is influenced by many external factors [1]-[3]. An example of an aggregate behavior may happen when relatively large group of consumers is turning on their home air conditioners within a short time span, as a consequence of a hot weather. This aggregated behavior is easy to notice since temperature increase affects a large population which might cause the peak. However, there are other factors that are likely to influence users' electrical consumption and there-fore, it is not trivial to foresee what will be the consumption level and, in turn, to detect high loads in advance.

Consumption peaks may cause serious challenges to electricity providers because they need to over-dimension the grid in order to support the abnormally high consumption load. Managing these peaks is crucial for the providers since energy scarcity can lead to severe consequences such as power outages. An alternative approach to overreach these peaks and to reduce the costs of over-dimensioning and enormity is to balance the grid with the introduction of intelligent methods for controlling them. Controlling the peaks can be done in several ways, such as performing load balancing and developing dynamic and intelligent pricing strategies taking into account that end users are sensitive to price and they may reduce the consumption whenever the electricity price is high.

The proposed paper is focused on detection of electrical power consumption peaks in the Polish power system by relying on historical data for both: electricity and weather conditions including temperature and humidity. The contribution to the research is twofold. First, we deal with peak detection as binary classification problem unlike to most legacy studies formulating the problem as time-series forecasting. Second, we propose a wide set of artificial neural network parameters to assure the problem is thoroughly tested for the benefit of precise classification. We further experimented with data from Polish power system, and were able to prove the high accuracy in peaks detection.

The rest of this paper is organized as follows. In the second section the literature review on similar problems is presented. The data characteristics and their mapping into binary classification problem is presented in section three. The fourth section deals with the experiments carried out and their results. The paper ends with concluding remarks in the last section.

\section{LITERATURE REVIEW ON SIMILAR PROBLEMS}

Forecasting the energy consumption and load demand peak has been intensively studied. In recent years, the extensive research stream of forecasting models was based on traditional algorithms including time series analysis, regression and grey models, as well as soft computing algorithms including genetic algorithms, fuzzy logic and other machine learning methods.

Time series models represent the future values based on previous observations. The models which are based on time series have many forms adequate for forecasting electricity consumption volume and peak demand load in the electrical grid. For instance, the problem of forecasting the monthly peak demand of electricity in north India was studied by Ghosh [4] who combined two different time series: a multiplicative Seasonal Auto-Regression Integrated Moving Average (SARIMA) and Holt-Winters multiplicative exponential smoothing. In turn, Mati et al. [5] used time 
series to forecast the electricity demand in Nigeria. GarciaAscanio and Mate [6] used the interval time series to forecast the monthly electricity consumption per hour in Spain.

Another way to predict the energy consumption is to use statistical regression models that correlate the power consumption with a number of influencing variables. Energy distribution companies often use regression analysis to forecast the variable (dependent) values based on one or more independent (predictor) variables. The relationship can be described using simple linear functions (e.g. linear regression) or large non parametric models like Gaussian and neural network. Simple linear, multiple linear, quadratic and exponential regression models are typically used to forecast short term load demand (usually five minutes to one week ahead) with hour by hour load data. The quantile regression is recommended to predict the peak electricity demand as well. Gibbons and Faruqui [7] developed a method that used quantile regression to model the daily peak demand, and subsequently used a loss function to estimate a quantile for annual peak prediction. In order to model system uncertainty, inexactness and random daily 15-minutes peak power demand at distribution trans-formers, Nazarko and Zalewski [8] used a fuzzy regression model expressing the correlation between substation peak load and other customer explanatory variables.

Lack of detailed data or limited dataset make difficulties in predicting future peak demand value which is critical for the dispatching center to handle current operations (shortterm forecasting) or to plan development and modernization of the power system (long-term forecasting). In such circumstances artificial neural networks (ANN) appear to be excellent technique to deal with noisy and incomplete data. ANN has been used to predict the hourly electricity consumption prediction model in Saudi Arabia [9], Nigerian Electrical Power System [10], the long-term demand of electricity in Turkey [11] and in Iran [12]. The multilayer perception model (MLP) to forecast the long-term energy consumption in Greece was applied by Ekonomou [13]. He compared the results of the model with those resulting from the support vector machine and the linear regression model. The applied model occurred very promising. Another comparative study among ARIMA, ANN and multiple linear regression (MLR) models was performed by Kandananond [14] who predicted long-term electricity demand in Thailand.

Lastly, it is worth mentioning that analysis of the last decade of electricity demand data in European countries shows trend that the peak demand (largest daily demand) throughout the year usually occurs in the winter, during the weeks before Christmas or in the summer [15]. Factors causing this increase in electricity demand include the cold weather (increased use of electrical heating devices) or prolonged period of abnormally hot weather (increased use air conditioners).

\section{DATASET CHARACTERISTICS}

\section{A. Load data}

This study was performed based on historical data representing energy consumption in Polish power system [16]. The data set included 70128 observations (hourly data) covering time span between January $1^{\text {st }}, 2008$ and December $31^{\text {st }}, 2015$. Time series of the power system load exhibit annual, weekly and daily seasonal cycles as shown in Fig. 1. The daily curves differ in shape depending on the day type (workday, Saturday, Sunday) and season. Fig. 2 shows a smooth profile shape with relatively little electricity consumption in the early morning, a clearly defined peak in the evening and a slightly smaller defined peak in the late morning.

Changes in the daily load shape and load level during the year are influenced by weather conditions including temperature, wind speed, cloud cover, humidity, precipitation and daylight hours. The weekly cycles are determined by workdays and holidays. The multiple seasonal cycles in the load time series as well as trend and nonstationarity in mean and variance have to be captured by a forecasting model. Electricity load when it is considered as time series cannot be modeled directly and additional treatments such as detrending, or decomposition are needed.

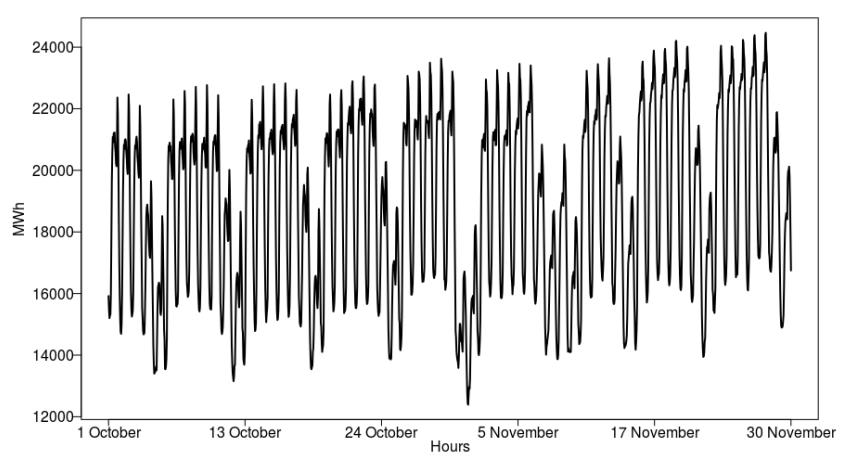

Fig. 1. Weekly load data covering time span between 1 st October 2015 and 30th November 2015

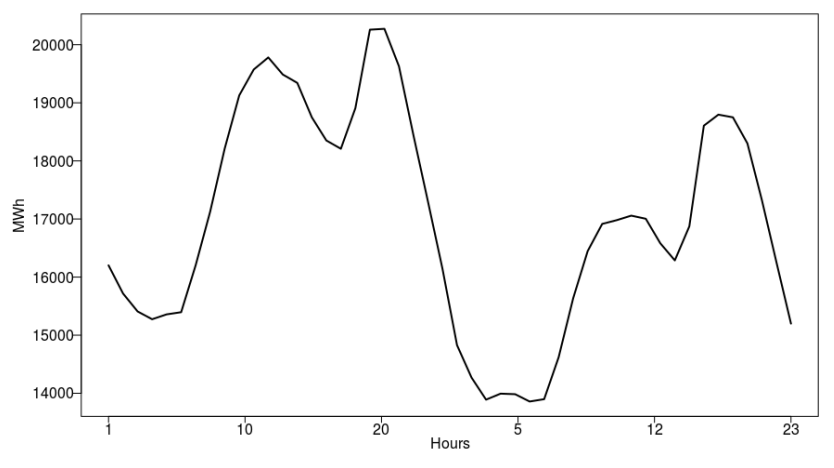

Fig. 2. Daily load data on October 23th (Friday) and $24^{\text {th }}$ (Saturday), 2015. 


\section{B. Weather data}

Weather is one of the most important independent variables for load forecasting, often described by temperature and humidity as presented in Fig. 3. The effect of weather is most prominent for domestic and agricultural consumers, but it can also alter the load profile of industrial consumers. Unexpected weather conditions are often cited as the tipping point that can cause unreliability in the system by decreasing the efficient supply of power. For instance, unpredicted thunderstorms in the middle of sunny day are one of the environmental factor that can decrease the temperature and thus causing overestimated load forecast [17], resulting in producing more power than required.

Temperature can also alter the conductivity of the transmission lines. Thus, it can affect the overall carrying capability of the transmission lines. High temperature can increase not only the resistance of the transmission lines, but also it can influence the reactance of line, as well as induced expansion of transmission line length [18].

There is a high positive correlation between temperature

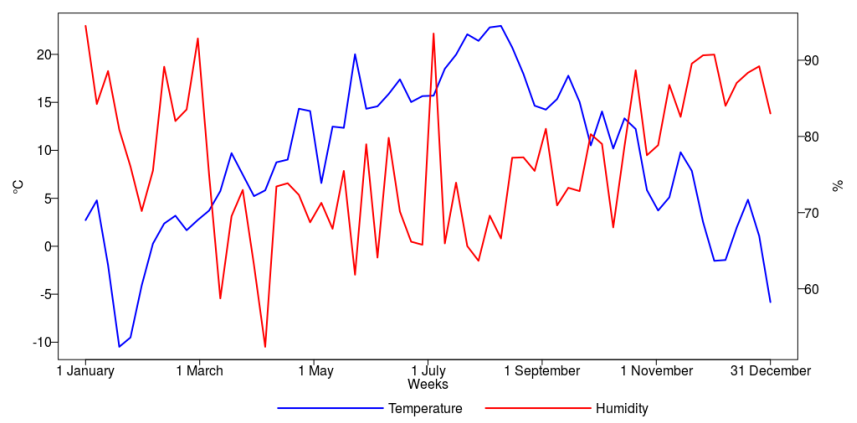

Fig. 3. Weekly average weather data in Warsaw in 2015

and load during summer season and there is a negative correlation between temperature and load during winter. This means that in the summer an increase in temperature will result in load increase whilst decrease in temperature will result in decrease in average daily load and also lowering the peak demand. In winter, the opposite trend is observed as decrease in per degree temperature will results in increase of electric load. This is because in summer increase in temperature affects consumers who use electricity for cooling purposes (air conditioners and fans), whereas in winter electricity is used for heating purposes. Hence, in winter there is negative or inverse relation between temperature and consumption volume [18].

Another weather factor influencing overall load is humidity. Formally, humid air was called not just the moist air but was referred as the mixture of water vapors and other constituents of air and humidity was defined in terms of water contents of this mixture called the absolute humidity [18]. In everyday life it is called relative humidity and is expressed in percentage. It is common observation that humidity can increase apparent temperature while it has no effect on the real temperature. This means humidity can make a $30^{\circ} \mathrm{C}$ temperature to be felt say $35^{\circ} \mathrm{C}$.

Although humidity has no effect on real temperature it can intensify the severity of hot climate. Therefore for the prediction of daily load at domestic consumers it is recommended to consider apparent temperature instead of real temperature. When dealing with mixed consumers, e.g. including industrial, agricultural and domestic, temperature humidity index can be employed as the factor influencing the load forecasting.

Finally, due to the high redundancy between weather and load data, a proper features selection approach in this research has to be considered.

\section{Determining peak values}

In order to determine peak load values, the generic function quantile was used [19]. The function produces sample quantiles corresponding to the given probabilities by the weighted averaging of order statistics $z_{g}$ :

$$
Q_{p}=(1-\gamma) z_{g}+\gamma z_{g+1},
$$

where $\gamma=n p+m-g, \quad n$ is number of observations, $g=$ floor $(n p+m)$ and $m=1-p$.

In this study, peak load was determined as the load value equal or above 99th percentile for a given load distribution when grouping load in each week, as presented in Fig. 4.

Black curve reflects real hourly electricity consumption observed in November 2015. Blue line shows average load within particular week, red line shows the behavior of threshold values above which the loads are recognized as peak values. Finally, green dots stand for peak load.

\section{NUMERICAL EXPERIMENT}

\section{A. Implementation and classification technique}

In our case, all the numerical calculations were performed

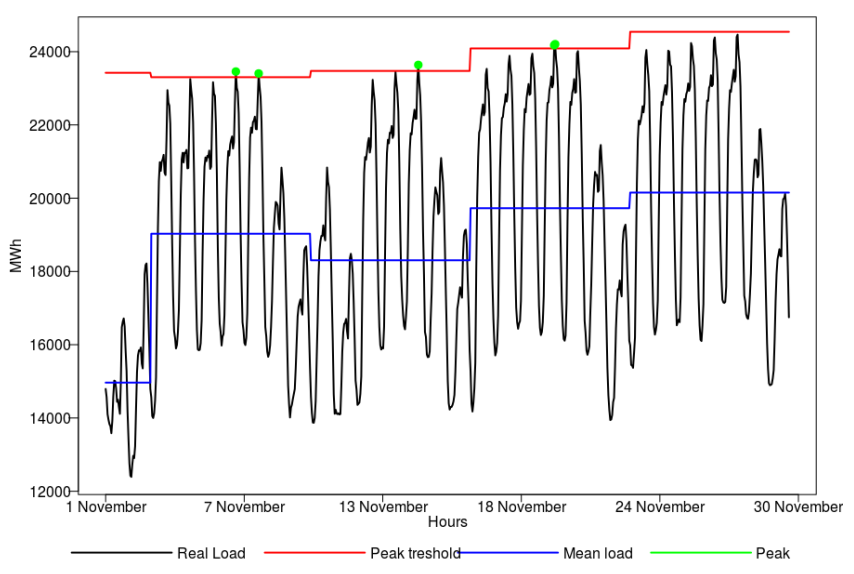

Fig. 4. Weekly peaks identification in the load data based on the 99th quantile of the load distribution (November 2015 data) 
on personal computer with the following parameters: Ubuntu 16.04 LTS operating system and Intel Core i5-2430M 2.4 GHz, 2 CPU* 2 cores, 8 GB RAM. R-CRAN [20], which is an advanced statistical package, as well as an interpreted programming language, was used as the computing environment. For training neural networks we used the BFGS algorithm, available in the nnet library [20]. A logistic function was used to activate all of the neurons in the neural network and initial vector with weights was chosen randomly using uniform distribution.

To compare the neural networks obtained for different number of hidden neurons we used the following measures: (1) AUC (area under the Receiver operating characteristic (ROC) curve), (2) classification accuracy, (3) Sensitivity (true positive rate), (4) Specificity (true negative rate). Those measures are related to efficiency and effectiveness of the ANN and they have been often used for evaluation of classification models in the context of various practical problems such as credit scoring, income and poverty determinants or customer insolvency and churn [21]-[24].

The dataset was split into three parts which corresponded to the training, validation and testing samples with the following proportion. The training sample consisted of 6 years between January $1^{\text {st }} 2008$, and December $31^{\text {st }} 2013$; the validation sample consisted of one year between January $1^{\text {st }}$ 2014, and December $31^{\text {st }} 2014$; and finally, the testing sample consisted also one year between January $1^{\text {st }} 2015$, and December $31^{\text {st }} 2015$.

The main criterion taken into account while learning the models is to gain good generalization of knowledge with the least error. The most commonly used measure to assess the quality of binary classification problem is AUC. Therefore, to find the best parameters for all models and to assure their generalization, the following function was maximized:

$$
f\left(A U C_{T}, A U C_{V}\right)=-\frac{1}{2}\left|A U C_{T}-A U C_{V}\right|+\frac{1}{2} A U C_{V}
$$

where $A U C_{T}$ and $A U C_{V}$ stand for the training and validation errors, respectively.

In contrast to other machine learning algorithms, ANN required special preparation of the input data. The vector of continuous variables has been standardized, while the binary variables were converted in a way that the value of 0 was transformed into -1 .

In the experiment we tried several neural network structures to get the best result. The number of neurons in hidden layer was proposed as a result of numerical procedure. We started neural network learning with small number of hidden units and then, successively, we increased number of neurons until no significant improvement in terms of models performance was observed (the number of neurons considered in the hidden layer was from 5 to 15). To avoid overfitting, after the completion of each learning iteration (with a maximum of 50 iterations - because already after 20 iterations the difference in terms of AUC between the learning and validation set began to increase), the models were checked for the error measure defined in equation (2). At the end, the ANN characterized by the smallest error was chosen as the best model. In order to achieve robust estimation of models' error, for each number of hidden neurons, ten different ANN were learned with different initial weights vector. Final estimation of the error was

TABLe 1.

FEATURE VECTOR USED FOR MODEL ESTIMATION

\begin{tabular}{ccc}
\hline $\begin{array}{c}\text { Attribute } \\
\text { No. }\end{array}$ & Description & Formula \\
\hline $1-5$ & Hour indicator (bits encoding) & $G_{i}, i=1, \ldots, 24$ \\
$6-10$ & Day of the month indicator (bits encoding) & $D_{i}, i=1, \ldots, 31$ \\
$11-13$ & Day of the week indicator (bits encoding) & $T_{i}, i=1, \ldots, 7$ \\
$14-17$ & Month indicator (bits encoding) & $M_{i}, i=1, \ldots, 12$ \\
18 & Holiday indicator (dummy variable) & $S$ \\
19 & Sunset indicator (dummy variable) & $N$ \\
$20-43$ & Load of previous 24 hours & $Z_{g-i}, i=1, \ldots, 24$ \\
$44-51$ & Average load observed over previous hourly intervals & $\operatorname{avg}\left\{W_{g-i}, \ldots, W_{g-i[+1]}\right\}, i=3,6,9,12,15,18,21,24$ \\
$52-57$ & Load in the same hour of the previous week & $Z_{g, d-i}, i=2, \ldots, 7$ \\
$58-65$ & Linear trend of the load observed over previous hourly periods & $\operatorname{Trend}\left\{W_{g-1}, \ldots, \mathrm{W}_{g-i}\right\}, i=3,6,9,12,15,18,21,24$ \\
$66-73$ & Average temperature observed over previous hours & $\operatorname{avg}\left\{T_{g-1}, \ldots, \mathrm{T}_{g-i}\right\}, i=3,6,9,12,15,18,21,24$ \\
$74-81$ & Average temperature observed over previous hourly intervals & $\operatorname{avg}\left\{T_{g-i}, \ldots, \mathrm{T}_{g-i[+1]}\right\}, i=3,6,9,12,15,18,21,24$ \\
$82-89$ & Average humidity observed over previous hours & $\operatorname{avg}\left\{H_{g-1}, \ldots, \mathrm{H}_{g-i}\right\}, i=3,6,9,12,15,18,21,24$ \\
$90-97$ & Average humidity observed over previous hourly intervals & $\operatorname{avg}\left\{H_{g-i}, \ldots, \mathrm{H}_{g-i[+1]}\right\}, i=3,6,9,12,15,18,21,24$ \\
\hline
\end{tabular}

Notation $[+1]$ stands for the next element from the set of indices $i\{1,3,6,12,24\}$ e.g. $\operatorname{avg}\left\{T_{g-1}, \ldots, T_{g-3}\right\}$ or $\operatorname{avg}\left\{T_{g-6}, \ldots, T_{g-9}\right\}$. Source: own preparation. 
computed as the average value over ten models and for each number of hidden neurons.

\section{B. Feature vector}

We focused on the next-day peak power demand detection. To forecast the peak, we constructed a feature vector with attributes as presented in Table 1. The attributes were constructed based on time series with hourly electricity demand. Additionally, other features were collected, including temperature, humidity, and calendar variables.

Electricity demand varies over time depending on the time of day (daily cycles), day of the week (weekly cycles), day of the month (monthly cycles), season (seasonal cycles) and occurrence of holidays. Therefore, we enriched the analysis with additional 18 variables including 5 variables describing the hour, 5 variables associated with the day of the month, 3 variables associated with the day of the week, 4 variables associated with the month, 1 variable indicating a holiday and 1 variable indicating the sunset in a particular hour. All above variables were derived in the following manner (bits encoding instead standard dummy encoding): first the categories were encoded as ordinal, then those integers were converted into binary code, then the digits from the binary string were split into separate columns. This encodes the data in fewer dimensions than standard dummy encoding.

The main variables taken into account in the forecasting process are those derived directly from the time series. The features were created by the decomposition of the time series, and they define, among others, linear trend and actual demand at certain intervals, taking into account up to 7 days of the history.

\section{Feature selection}

In order to identify dependence between observed peak load and explanatory variables Kolmogorov-Smirnov statistics was applied as presented in Table 2.

The Kolmogorov-Smirnov statistic quantifies a distance between the empirical distribution function of the sample and the cumulative distribution function of the reference distribution, or between the empirical distribution functions of two samples. In our case we derived two samples, i.e. distribution for peak cases and distribution for non-peak cases. Based on the obtained p-values it was identified that most of the considered features have different distributions within positive and negative cases. The only exceptions are observed for the following variables $(0.05$ statistical significance assumed): temp_1_15, temp_1_18, hum_1_21, hum_1_24 and hum_avg_19_21.

Next, the Chi2 $\left(\chi^{2}\right)$ test was used to determine whether there is a significant difference between the expected and the observed frequencies in one or more categories of each independent variable and dependent variable. Each independent variable was divided into 10 disjoint groups based on the quantiles values of the particular distribution (based on deciles). Then each feature and dependent variable have created a contingency table $2 \times 10$. Finally, based on the aforementioned table, Chi2 test was applied.

Proposed test showed that there is statistically significant dependence between independent variable and all dependent variables. On the other hand, in case of the categorical features, variables indicating holiday days and day of the month were not statistically significant (please refer to Table 3 for details).

The third approach to determine appropriate set of

TABLE 2 .

THE RESULTS OF THE KOLMOGOROV-SMIRNOV STATISTICS FOR QUANTITATIVE VARIABLES

\begin{tabular}{|c|c|c|c|c|c|c|c|}
\hline Variable name & $\begin{array}{c}K-S \\
p \text {-value }\end{array}$ & Variable name & $\begin{array}{c}K-S \\
p-v a l u e\end{array}$ & Variable name & $\begin{array}{c}K-S \\
p \text {-value }\end{array}$ & Variable name & $\begin{array}{c}K-S \\
\text { p-value }\end{array}$ \\
\hline $\mathrm{t} \_1$ & 0.0000 & t_21 & 0.0000 & d_2 & 0.0000 & temp_avg_19_21 & 0.0005 \\
\hline t_2 & 0.0000 & t_22 & 0.0000 & d_3 & 0.0000 & temp_avg_22_24 & 0.0000 \\
\hline t_3 & 0.0000 & t_23 & 0.0000 & d_4 & 0.0000 & hum_avg_1_3 & 0.0000 \\
\hline t_4 & 0.0000 & t_24 & 0.0000 & d_5 & 0.0000 & hum_avg_4_6 & 0.0000 \\
\hline $\mathrm{t} \_5$ & 0.0000 & temp_1_3 & 0.0000 & d_6 & 0.0000 & hum_avg_7_9 & 0.0000 \\
\hline t_6 & 0.0000 & temp_1_6 & 0.0000 & d_7 & 0.0000 & hum_avg_10_12 & 0.0000 \\
\hline t_7 & 0.0000 & temp_1_9 & 0.0000 & avg_1_3 & 0.0000 & hum_avg_13_15 & 0.0000 \\
\hline t_8 & 0.0000 & temp_1_12 & 0.0022 & avg_4_6 & 0.0000 & hum_avg_16_18 & 0.0000 \\
\hline t_9 & 0.0000 & temp_1_15 & 0.1550 & avg_7_9 & 0.0000 & hum_avg_19_21 & 0.1383 \\
\hline $\mathrm{t} \_10$ & 0.0000 & temp_1_18 & 0.0718 & avg_10_12 & 0.0000 & hum_avg_22_24 & 0.0001 \\
\hline t_11 & 0.0000 & temp_1_21 & 0.0303 & avg_13_15 & 0.0000 & trend_1_3 & 0.0000 \\
\hline $\mathrm{t} \_12$ & 0.0000 & temp_1_24 & 0.0229 & avg_16_18 & 0.0000 & trend_1_6 & 0.0000 \\
\hline t_13 & 0.0000 & hum_1_3 & 0.0000 & avg_19_21 & 0.0000 & trend_1_9 & 0.0000 \\
\hline t_14 & 0.0000 & hum_1_6 & 0.0000 & avg_22_24 & 0.0000 & trend_1_12 & 0.0000 \\
\hline $\mathrm{t} \_15$ & 0.0000 & hum_1_9 & 0.0000 & temp_avg_1_3 & 0.0000 & trend_1_15 & 0.0000 \\
\hline t_16 & 0.0000 & hum_1_12 & 0.0000 & temp_avg_4_6 & 0.0000 & trend_1_18 & 0.0000 \\
\hline t_17 & 0.0000 & hum_1_15 & 0.0001 & temp_avg_7_9 & 0.0489 & trend_1_21 & 0.0000 \\
\hline t_18 & 0.0000 & hum_1_18 & 0.0191 & temp_avg_10_12 & 0.0007 & trend_1_24 & 0.0000 \\
\hline t_19 & 0.0000 & hum_1_21 & 0.1227 & temp_avg_13_15 & 0.0000 & & \\
\hline t_20 & 0.0000 & hum_1_24 & 0.1610 & temp_avg_16_18 & 0.0000 & & \\
\hline
\end{tabular}


TABLE 3.

THE RESULTS OF THE CHI2 STATISTICS FOR THE CATEGORICAL VARIABLES

\begin{tabular}{cc}
\hline Variable name & $\begin{array}{c}\text { Chi2 } \\
\text { p-value }\end{array}$ \\
\hline month & 1.0000 \\
month_day & 0.0977 \\
hour & 0.0000 \\
week_day & 0.0000 \\
holiday & 1.0000 \\
sunset & 0.0000 \\
\hline
\end{tabular}

Source: own preparation.

independent variables was Area Under the ROC curve (AUC). In this case discriminatory power of each variable was check out in the following manner:

- quantitative and ordinal variable were sorted in ascending order; categorical variables were sorted in ascending order based on the conditional probability of belonging into positive cases.

- ROC curve was determined. The actual values of the sorted variable served as the score values of the classification model.

- AUC measure was computed using trapezoidal integration.

Final AUC values for all the features are presented in Table 4 (quantitative variables) or Table 5 (categorical variables).

In the case of quantitative variables, the greatest discriminatory power can be assigned to: d_6, d_5, trend_1_18, trend_1_15, d_7, trend_1_3 and t_1 attributes. Out of categorical variables, two of them - hour and week days - have the best performance.

Obviously, there is a strictly linear dependence between some features, which means that the redundancy in the data could be observed. There is no need to include for instance variable t 4 and t 5 in final input vector, due to collinearity. Therefore, from the best set of attributes, the variables having Spearman correlation coefficient greater than 0.6 were removed. Eventually, the final set of attributes is presented in Table 6 .

\section{Results}

In order to benefit from the optimal score threshold which determine the peak (score above the threshold) or normal load (score below the threshold), Youden's J statistic [25] was employed. The optimal cut-off is the threshold that maximizes the distance to the identity (diagonal) line. The optimality criterion is defined as:

$$
\max (\text { sensitivities }+ \text { specifitities })
$$

In this research optimal cut-off was identified at 0.1111 .

The classification results obtained on training, validation and testing datasets are presented in Table 7 (upper and lower part). Importantly, the models exhibited stable performance in terms of the classification quality on all three datasets. For the testing sample the accuracy, which measures of how many correct forecasts the model makes, is up to $90.5 \%$, and this is observed for the neural networks with six and eleven hidden neurons. The AUC measure for the models is ranging between 0.947 and 0.967 . In terms of the sensitivity, which is proportion of peaks that are correctly identified as such, the results are ranging between 0.915 and 0.962 . Finally, specificity which measures the proportion of non-peaks that are correctly identified as such is ranging from 0.855 to 0.904 .

Taking into account that simpler model should be preferred over the complex one, the neural network with 9

TABLE 4.

AUC VALUES FOR THE QUANTITATIVE VARIABLES

\begin{tabular}{|c|c|c|c|c|c|c|c|}
\hline Variable name & $A U C$ & Variable name & $A U C$ & Variable name & $A U C$ & Variable name & $A U C$ \\
\hline t_1 & 0.744 & t_21 & 0.551 & d_2 & 0.677 & temp_avg_19_21 & 0.53 \\
\hline t_2 & 0.678 & t_22 & 0.629 & d_3 & 0.673 & temp_avg_22_24 & 0.496 \\
\hline t_3 & 0.663 & t_23 & 0.689 & d_4 & 0.74 & hum_avg_1_3 & 0.615 \\
\hline t_4 & 0.665 & t_24 & 0.72 & d_5 & 0.815 & hum_avg_4_6 & 0.65 \\
\hline t_5 & 0.644 & temp_1_3 & 0.528 & d_6 & 0.84 & hum_avg_7_9 & 0.557 \\
\hline t_6 & 0.608 & temp_1_6 & 0.54 & $d_{-}^{-7}$ & 0.766 & hum_avg_10_12 & 0.556 \\
\hline t_7 & 0.56 & temp_1_9 & 0.532 & avg_1_3 & 0.701 & hum_avg_13_15 & 0.61 \\
\hline $\mathrm{t} \_8$ & 0.531 & temp_1_12 & 0.514 & avg_4_6 & 0.641 & hum_avg_16_18 & 0.582 \\
\hline t_9 & 0.514 & temp_1_15 & 0.504 & avg_7_9 & 0.535 & hum_avg_19_21 & 0.508 \\
\hline t_10 & 0.514 & temp_1_18 & 0.514 & avg_10_12 & 0.575 & hum_avg_22_24 & 0.552 \\
\hline t_11 & 0.573 & temp_1_21 & 0.516 & avg_13_15 & 0.709 & trend_1_3 & 0.752 \\
\hline $\mathrm{t} \_12$ & 0.638 & temp_1_24 & 0.514 & avg_16_18 & 0.688 & trend_1_6 & 0.632 \\
\hline t_13 & 0.687 & hum_1_3 & 0.615 & avg_19_21 & 0.529 & trend_1_9 & 0.657 \\
\hline t_14 & 0.714 & hum_1_6 & 0.638 & avg_22_24 & 0.684 & trend_1_-12 & 0.725 \\
\hline $\mathrm{t} \_15$ & 0.708 & hum_1_9 & 0.619 & temp_avg_1_3 & 0.528 & trend_1_15 & 0.786 \\
\hline t_16 & 0.696 & hum_1_12 & 0.578 & temp_avg_4_6 & 0.549 & trend_1_18 & 0.788 \\
\hline t_17 & 0.69 & hum_1_15 & 0.54 & temp_avg_7_9 & 0.509 & trend_1_21 & 0.731 \\
\hline t_18 & 0.664 & hum_1_18 & 0.517 & temp_avg_10_12 & 0.543 & trend_1_24 & 0.607 \\
\hline t_19 & 0.608 & hum_1_21 & 0.513 & temp_avg_13_15 & 0.571 & & \\
\hline t_20 & 0.53 & hum_1_24 & 0.519 & temp_avg_16_18 & 0.564 & & \\
\hline
\end{tabular}

Source: own preparation. 
TABLE 5.

AUC VALUES FOR THE CATEGORICAL VARIABLES

\begin{tabular}{cc}
\hline Variable name & AUC \\
\hline month & 0.504 \\
month_day & 0.509 \\
hour & 0.748 \\
week_day & 0.678 \\
\hline \multicolumn{2}{c}{ Source: own preparation. }
\end{tabular}

hidden neurons represents fair tradeoff between the complexity and the classification quality.

Additionally, to give also a graphical view on the performance of the proposed model with 9 neurons, one dayahead peak forecast obtained for the randomly drawn test period (five weeks in October 2015) is shown in Fig. 5. From the figure we can observe that the peak loads are correctly predicted in seven cases - green dots represent true positive classification. Three peak loads, marked as red dots, are incorrectly classified as a normal loads (false negative classification). Finally, in some cases (yellow dots), neural network claims that there will be peak load in one day ahead, but actually there was no peak (false positive classification). For the clarity of the Fig. 5 True negative class was not provided as it constitutes for the overwhelming majority.

The results of the numerical experiments can be summarized as follows:

- Peak demands in Poland are mostly affected by such features as day of the week, temperature, humidity, load in previous hours and the load trend observed in previous hours;

- The best results were obtained for the neural network with 9 hidden neurons;

- Predictive power of the model is considered to be excellent what was confirmed by AUC, accuracy, sensitivity and specificity measures;

- High true positive rate confirms the models ability to correctly classify the real peaks in the system.

TABLE 6.

THE FINAL SET OF ATTRIBUTES

\begin{tabular}{c}
\hline Variable name \\
\hline avg_1_3 \\
d_4 \\
d_6 \\
hour \\
hum_avg_4_6 \\
hum_avg_13_15 \\
t_14 \\
t_18 \\
t_24 \\
trend_1_3 \\
trend_1_12 \\
trend_1_18 \\
week_day \\
\hline
\end{tabular}

Source: own preparation.

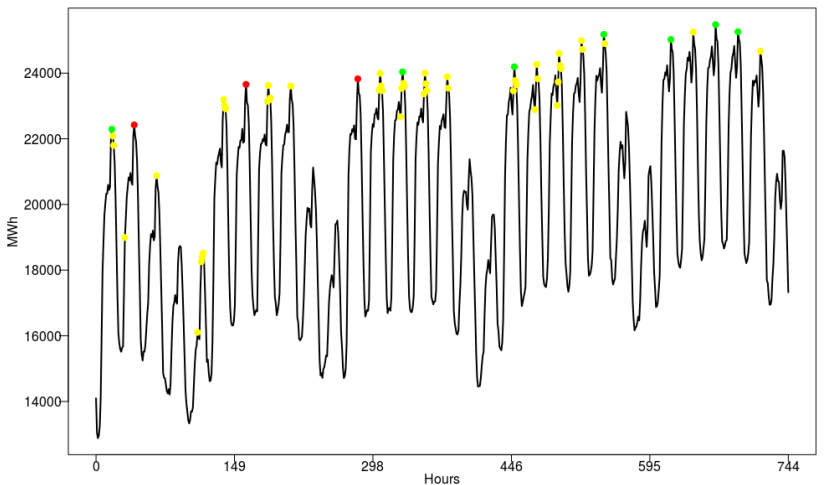

Fig. 5. Prediction results in October 2015. Color denotes the result of the classification as follows: True positive - green, False negative - red, False positive - yellow.

\section{SUMMARY AND CONCLUDING REMARKS}

The research addresses the problem of predicting electrical consumption peaks as an input into load balancing and smart pricing strategies. This was done by mapping the problem into a binary classification task aimed to detect the peaks using the features based on previous consumption and the weather data.

The contribution of this study provides the proof that models can capture the complex nonlinear effects of historical load, temperature, humidity and calendar effects. The classification results demonstrate that neural networks models perform remarkably well on the historical data.

The most promising results were produced by applying artificial neural network with 9 hidden neurons what led to predicting $96.2 \%$ of the true peaks (sensitivity) as observed on the testing dataset. It is worth mentioning that the algorithm was to favor false positives over false negatives as the latter are having less impact on electrical power grids. This is because a false positive, which is predicting a peak that is not present, has significantly fewer consequences than not predicting peaks which are present. Thus, high true positive rate is much more favored after than high precision of the model.

There are number of practical applications to make use of next-day peak power demand identification. Forecasts of the peak demand are useful for both, network capacity planning and investment decisions. In addition, the knowledge on the timing of the peak demand is important for network maintenance planning. An accurate classification can be used to improve decision making and the correct classification can reduce both costs and risks for the entities operating on the electricity markets.

\section{ACKNOWLEDGMENT}

The study was cofounded by the National Science Centre, Poland, Grant No. 2016/21/N/ST8/02435. 
TABLE 7.

CLASSIFICATION RESULTS FOR THE NEXT-DAY PEAK POWER DEMAND

Upper part

\begin{tabular}{|c|c|c|c|c|c|c|c|c|c|c|}
\hline \multirow{2}{*}{$\begin{array}{c}\text { Number } \\
\text { of hidden } \\
\text { neurons }\end{array}$} & \multirow{2}{*}{$\begin{array}{c}\text { Average } \\
\text { number of } \\
\text { iterations }\end{array}$} & \multicolumn{4}{|c|}{ Training sample } & \multicolumn{4}{|c|}{ Validation sample } & \multirow{2}{*}{$\begin{array}{c}\text { AUC Equation } \\
\text { No } 2\end{array}$} \\
\hline & & Accuracy & $\boldsymbol{A U C}$ & Sensitivity & Specificity & Accuracy & $\boldsymbol{A U C}$ & Sensitivity & Specificity & \\
\hline 5 & 27.0 & 0.912 & 0.964 & 0.933 & 0.912 & 0.868 & 0.954 & 0.95 & 0.867 & 0.944 \\
\hline 7 & 27.8 & 0.9 & 0.968 & 0.955 & 0.899 & 0.884 & 0.959 & 0.956 & 0.883 & 0.95 \\
\hline 8 & 25.2 & 0.906 & 0.973 & 0.958 & 0.906 & 0.888 & 0.963 & 0.956 & 0.887 & 0.953 \\
\hline 9 & 26.0 & 0.91 & 0.975 & 0.957 & 0.909 & 0.903 & 0.967 & 0.947 & 0.903 & 0.96 \\
\hline 11 & 20.4 & 0.907 & 0.972 & 0.95 & 0.907 & 0.891 & 0.966 & 0.962 & 0.89 & 0.961 \\
\hline 12 & 21.2 & 0.872 & 0.957 & 0.945 & 0.872 & 0.858 & 0.944 & 0.941 & 0.857 & 0.932 \\
\hline 13 & 23.0 & 0.892 & 0.965 & 0.948 & 0.891 & 0.862 & 0.958 & 0.966 & 0.861 & 0.951 \\
\hline 14 & 23.4 & 0.899 & 0.969 & 0.957 & 0.899 & 0.87 & 0.962 & 0.981 & 0.869 & 0.956 \\
\hline 15 & 22.0 & 0.889 & 0.962 & 0.937 & 0.889 & 0.85 & 0.954 & 0.966 & 0.849 & 0.946 \\
\hline
\end{tabular}

Lower part

\begin{tabular}{ccccc}
\hline \multirow{2}{*}{$\begin{array}{c}\text { Number of } \\
\text { hidden } \\
\text { neurons }\end{array}$} & Accuracy & AUC & Sensitivity & Specificity \\
\cline { 2 - 5 } & 0.88 & 0.958 & 0.944 & 0.88 \\
5 & 0.905 & 0.956 & 0.915 & 0.904 \\
6 & 0.884 & 0.958 & 0.94 & 0.883 \\
7 & 0.886 & 0.963 & 0.95 & 0.885 \\
8 & 0.893 & 0.967 & 0.962 & 0.892 \\
9 & 0.856 & 0.948 & 0.94 & 0.855 \\
10 & 0.905 & 0.963 & 0.933 & 0.904 \\
11 & 0.863 & 0.947 & 0.938 & 0.862 \\
12 & 0.882 & 0.957 & 0.938 & 0.881 \\
13 & 0.868 & 0.96 & 0.962 & 0.867 \\
14 & 0.865 & 0.954 & 0.946 & 0.864 \\
15 & & & &
\end{tabular}

Source: own preparation.

\section{REFERENCES}

[1] M. Goodwin, and A. Yazidi, "A Pattern Recognition Approach for Peak Prediction of Electrical Consumption". In L. Iliadis, I. Maglogiannis, H. Papadopoulos (Eds) Proc. Artificial Intelligence Applications and Innovations AIAI 2014, IFIP Advances in Information and Communication Technology, vol. 436, Springer, Berlin Heidelberg, 2014, http://dx.doi.org/10.1007/978-3-662-44654626

[2] A. Goia, C. May, and G. Fusai, "Functional clustering and linear regression for peak load forecasting", International Journal of Forecasting, vol. 26, no. 4, 2010, pp. 700-711, http://dx.doi.org/10.1016/j.ijforecast.2009.05.015

[3] E. Chiodo, and D. Lauria, "Probabilistic description and prediction of electric peak power demand", Electrical Systems for Aircraft, Railway and Ship Propulsion (ESARS) IEEE, 2012. pp. 1-7, http://dx.doi.org/10.1109/ESARS.2012.6387418

[4] S. Ghosh, "Univariate time-series forecasting of monthly peak demand of electricity in northern India", International Journal of Indian Culture and Business Management, vol. 1, no. 4, 2008, pp. 466-474, http://dx.doi.org/10.1504/IJICBM.2008.018626

[5] A. A. Mati, B. G. Gajoga, B. Jimoh, A. Adegobye, and D. D. Dajab, "Electricity demand forecasting in Nigeria using time series model", The Pacific Journal of Science and Technology, vol. 10, no. 2, 2009, pp. $479-485$.

[6] C. García-Ascanio, and C. Maté, "Electric power demand forecasting using interval time series: A comparison between VAR and iMLP",
Energy Policy, vol. 38, no. 2, 2010, pp. 715-25, http://dx.doi.org/10.1016/j.enpol.2009.10.007

[7] C. Gibbons, and A. Faruqui, "Quantile Regression for Peak Demand Forecasting”, Available at SSRN 2485657, 2014 Jul 31, http://dx.doi.org/10.2139/ssrn.2485657

[8] J. Nazarko, and W. Zalewski, "The Fuzzy Regression Approach to Peak Load Estimation in Power Distribution Systems", IEEE Transactions on Power Systems, vol. 14, no. 3, 1999, http://dx.doi.org/10.1109/59.780890

[9] A. J. Al-Shareef, E. A. Mohamed, and E. Al-Judaibi, "Next 24-Hours Load Forecasting using Artificial Neural Network (ANN) for the Western Area of Saudi Arabia”, J. Faculty of Eng. Sci, King Abdulaziz University (KAU), vol.19, no. 2, 2008, pp. 25-40.

[10] G. A., Adepoju, S. O. Ogunjuyigbe, and K. O. Alawode, "Application of Neural Network to Load Forecasting in Nigerian Electrical Power System", The Pacific Journal of Science and Technology, Akamai University, vol. 8, no. 1, 2007, pp. 68-72.

[11] M. Çunkaş, and A. A. Altun, "Long term electricity demand forecasting in Turkey using artificial neural networks", Energy Sources, Part B: Economics, Planning, and Policy, vol. 5, no. 3, 2010, pp. 279-289.

[12] L. Ghods, and M. Kalantar, "Long-term peak demand forecasting by using radial basis function neural networks", Iranian Journal of Electrical and Electronic Engineering, vol. 6, no. 3, 2010, pp. 175182.

[13] L. Ekonomou, "Greek long-term energy consumption prediction using artificial neural networks", Energy, vol. 35, no. 2, 2010, pp. 512-517, http://dx.doi.org/10.1016/j.energy.2009.10.018 
[14] K. Kandananond, "Forecasting electricity demand in Thailand with an artificial neural network approach", Energies, vol. 4, no. 8, 2011, pp. 1246-1257, http://dx.doi.org/10.3390/en4081246

[15] P. E. McSharry, S. Bouwman, and G. Bloemhof, "Probabilistic forecasts of the magnitude and timing of peak electricity demand". IEEE Transactions on Power Systems, vol. 20, no. 2, 2005, pp. 11661172, http://dx.doi.org/10.1109/TPWRS.2005.846071

[16] Polish power system dataset, http://www.pse.pl/index.php?dzid=77, accessed 2016/08/12.

[17] S. Rahman, "Formulation and analysis of a rule-based short-term load forecasting algorithm", Proc. of IEEE, vol. 78, no. 5, 1990, pp. 805816, http://dx.doi.org/10.1109/5.53400

[18] M. U. Fahad, and N. Arbab, "Factor Affecting Short Term Load Forecasting", Journal of Clean Energy Technologies, vol. 2, no. 4, 2014, pp. 305-309, http://dx.doi.org/10.7763/JOCET.2014.V2.145

[19] R. J. Hyndman, and Y. Fan, "Sample quantiles in statistical packages", American Statistician, vol. 50, no. 4, 1996, pp. 361-365, http://dx.doi.org/10.2307/2684934

[20] R Core Team: A language and environment for statistical computing, R Foundation for Statistical Computing, Vienna, Austria, 2015.
[21] K. Gajowniczek, T. Ząbkowski, and R. Szupiluk, "Estimating the ROC curve and its significance for classification models' assessment", Quantitative Methods in Economics, vol. 15, no. 2, 2014, pp. 382391.

[22] K. Gajowniczek, T. Ząbkowski, and A. Orłowski, "Entropy Based Trees to Support Decision Making for Customer Churn Management", Acta Physica Polonica A, vol. 129, no. 5, 2016, pp. 971-979, http://dx.doi.org/10.12693/APhysPolA.129.971

[23] K. Gajowniczek, K. Karpio, P. Łukasiewicz, A. Orłowski, and T. Ząbkowski, "Q-entropy approach to selecting high income households", Acta Physica Polonica A, vol. 127, no. 3A, 2015, pp. 38-44, http://dx.doi.org/10.12693/APhysPolA.127.A-38

[24] K. Gajowniczek, T. Ząbkowski, and A. Orłowski, "Comparison of Decision Trees with Renyi and Tsallis Entropy Applied for Imbalanced Churn Dataset", Annals of Computer Science and Information Systems, vol. 5, 2015, pp. 39-43, http://dx.doi.org/10.15439/2015F121

[25] W. J. Youden, "An index for rating diagnostic tests", Cancer, vol. 3, 1950, pp. 32-35, http://dx.doi.org/10.1002/10970142(1950)3:1\%3C32::AID-CNCR2820030106\%3E3.0.CO;2-3 